\title{
Autonomous Charging of Electric Vehicles in Industrial Environment
}

\author{
Mario Hirz*, Bernhard Walzel, Helmut Brunner
}

\begin{abstract}
Modern industrial manufacturing involves several manually and automated driven vehicles - not only for logistics and production purposes, but also for services maintenance, resources supply and cleaning. These different types of vehicles are increasingly driven by electric powertrains that operate in the production halls, warehouses and other involved areas. Today, electric charging of these mobile devices is accomplished mainly manually and by use of a number of different not standardized charging interfaces, which leads to increased time and cost efforts. The paper evaluates different charging technologies for the use in industrial environments and introduces a new approach for automated, robot-controlled charging of electric vehicles, which is based on a standardized charging interface. The technology has been developed to fully automated charge different types of cars and other vehicles and consists of a vision system to identify the vehicle and the charging connector position in combination with a fully-controlled robotic system that plugs-in and -off the charging connector. In this way, the system is universally applicable for different types of autonomously and manually driven vehicles in a professional context, e.g. in production, logistics and warehouses.
\end{abstract}

Keywords: automated charging; autonomous driving; electric vehicles; industrial services; robotics

\section{INTRODUCTION}

Electric propulsion systems in mobile industrial applications have a long tradition and can be found in multifarious types of use. Fig. 1 shows selected examples of electrically driven vehicles as they are applied in logistics, manufacturing plants and other types of professional facilities. Besides transportation systems, e.g. fork lifters, logistics manipulators and floor-borne vehicles, further applications are common, e.g. cleaning, maintenance and service inspection.
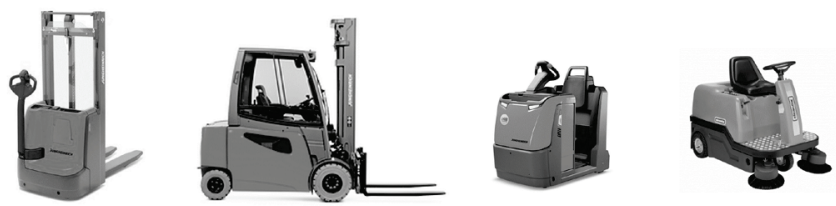

Figure 1 Exemplary manually driven electric vehicles for professional applications

$[1,2]$

In the past years, robotic systems are increasingly used in industry and logistics, e.g. for moving materials and components, in warehouses and also for services and cleaning. Fig. 2 shows a selection of fully automated vehicles for industrial use.

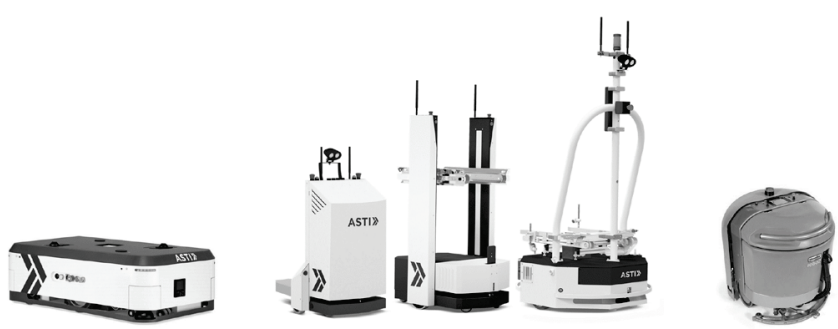

Figure 2 Selection of autonomous industrial robots [2, 3]

Regardless of manually or autonomously driven, the powertrain of such vehicles includes an electric energy storage system, typically based on high voltage lithium-ion technology, power electronics, electric motor(s) and a vehicle controller, an electric converter for charging, and eventually wireless data communication. As a status of today, the vehicles are charged by manually plugging-in charging cables or by use of type-specific charging sockets, being connected at a defined vehicle parking position. Especially for self-driving vehicles, manual charging represents a very non-practical approach, but also human-driven vehicles would benefit from a universally applicable, automated charging solution. Previously used company- and vehicle type-specific charging sockets come with the disadvantage of non-universal applicability and these systems are not certified for general use. In this context, it is a target of research and development activities to provide a universally applicable fully-automated charging solution that makes use of a robust and standardized charging interface. One of such solution has recently been introduced by the company Cleanfix [4]. This system combines a standardized charging interface from the automotive industry with a robotic arm to charge an autonomous floor-cleaning vehicle. In addition, services can be provided during the charging process, e.g. draining of waste water and refilling of fresh water for the next cleaning sequence.

Robotic service vehicles in industrial applications only make sense in combination with fully autonomous charging technology. And even for manually driven vehicles, the use of automated charging has a great potential to support safe operation and efficient processes. In this context, the present publication introduces results of a research project, which was focused on the development of a fully-automated charging system for a broad range of applications [5].

\section{OVERVIEW OF ELECTRIC VEHICLES CHARGING TECHNOLOGIES}

Various techniques enable electric charging of vehicles. In the following, the three most important charging principles with potential for automation are presented: conductive charging, inductive charging and battery swapping. 


\subsection{Inductive Charging}

Inductive charging enables wireless power transfer and is derived from the basic principle of a transformer, where energy transfer is provided via two oppositely positioned coils. Today, the technology is common in different consumer products with relatively low transfer power, e.g. smart phones or toothbrushes. In the case of vehicle charging, higher charging power is required, which arises several challenges. The arrangement principle of inductive charging systems for vehicles is similar to those of consumer product solutions: a primary coil is stationary installed at the charging station and a secondary coil is mounted at the vehicle's underbody. Advantages of this technology include contactless charging and high comfort, because no manual operation is necessary for connection. Furthermore, there is a low risk of unintended damage or vandalism because the system does not involve freely movable cables - just flat plates at the station and in the vehicle. Weaknesses of inductive charging are based on the electric principle of inductive power transfer. In this way, charging efficiency decreases with the size of the air gap between the coils and potential deviations of centric positioning of the charging pads. In addition, electromagnetic radiation is produced, which can lead to problems regarding electromagnetic compatibility (EMC). While progress in research and standardization is done towards higher charging power for automotive applications, maximal charging power is restricted to $3.6 \mathrm{~kW}$ today because of both EMC and the limited size of the charging pads. Nevertheless, there are a few applications in the automotive industry, e.g. introduced by BMW, Fig. 3 [6]. Because of limited charging power, issues with highly accurate positioning and relatively high system costs, inductive charging has not been considered as an option for an industrial applicable automated charging system in the course of the present research project.

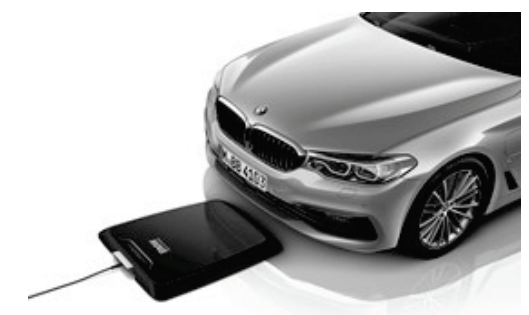

Figure 3 Serial application of inductive charging of cars [6]

\subsection{Battery Swapping Systems}

In battery swapping stations, the empty battery is decoupled from the vehicle and replaced by a fully charged battery unit. The battery exchange process takes just some minutes and the vehicle is ready to go. In the station, the battery can be recharged, checked for proper functionality and then provided to be implemented into another vehicle. In this way, battery swapping stations seem to be an attractive alternative to fixed mounted vehicle-internal batteries. The technology has been tested in the automotive industry since several years, e.g. by the company Better Place in a joint project with Renault [7]. A Chinese car manufacturer, Nio, has introduced battery swapping in mass production cars recently and also provides a number of battery swapping stations in large cities and on main highways, Fig. 4 [8]. Challenges and limitations of this technology are characterized by the relatively high investment to make the system running. Besides an automated robot-controlled battery swapping mechanism, a number of pre-charged batteries have to be provided to enable uninterrupted operation of the system. In addition, the vehicles have to be prepared for battery swapping, which requires stiff vehicle structures as well as a specific design of battery mounting and connecting systems in the cars. Finally, the battery swapping system has to manage and provide different battery sizes and formats to support all vehicle types involved. Because of these limitations - especially the high investment costs and the limited variability in view of servicing different vehicle types with varying battery sizes, this technology has not been considered as an option for an industrial applicable automated charging system in the course of the present research project.

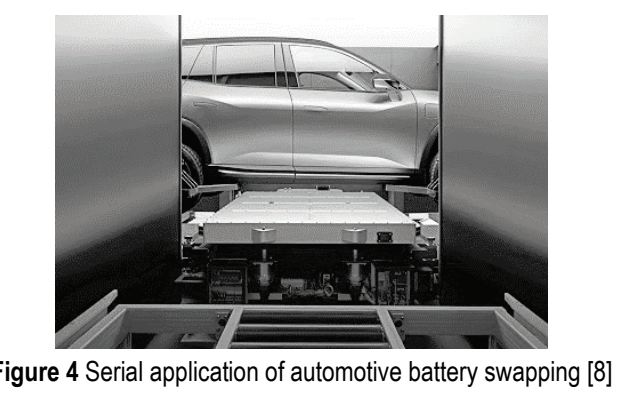

\subsection{Conductive Charging}

Conductive charging is characterized by direct electric connection by use of cables or specifically designed conducting elements. Due to the direct flow of electricity, high power can be transferred with very good efficiency. In this way, modern conductive charging systems enable up to $43 \mathrm{~kW}$ alternating current (AC) and $500 \mathrm{~kW}$ direct current (DC) charging power. Once the connection is established, the power transfer is safe and reliable. In general, conductive charging technologies distinguish between underbody coupler and so-called side-coupler. A special case represent pantograph systems with coupling over the vehicle's roof. This technology is well-known in railway systems and now under development for heavy truck and bus applications. Due to the large size of the pantograph systems, they are not considered further in the present work.

\subsubsection{Conductive Underbody Coupler}

Underbody coupler consist of a movable or fixed charging device that is placed on the floor of the charging station and a movable or fixed opposite unit mounted on the underbody of the vehicle. Entering the charging station, the vehicle is placed in a way that both units are aligned, whereas parking misalignments of about $0.5 \times 0.5 \mathrm{~m}$ are allowed. 
Connection is established by the motion of a connector at the movable unit onto the fixed unit. Fig. 5 exemplary shows two underbody coupling systems: The matrix-charging system from Easelink [9] has a moveable trunk mounted in the vehicle, which "screws" down to a fixed plate on the floor for contacting. The Volterio system does it the other way around: a robotic arm, mounted on the floor, moves up to a fixed connector in the vehicle [10]. Underbody couplers have the advantage of robustness and safe operation, because there are no cables hanging around. However, the integration effort is relatively high. In case of technologies with moveable stationary units, the robotic kinematics and control is complex, and in case of moveable units in the vehicles, the vehicle integration requires installation space and complex control of the kinematics. These aspects might be the reason why underbody coupler technologies have not been brought into mass production vehicles so far. In case of professional use in industrial environment, underbody coupler cannot be used for some specific vehicle types, e.g. flat manipulation robots or cleaning machines. For this reason, they are not considered further as a potential technology in the present project.
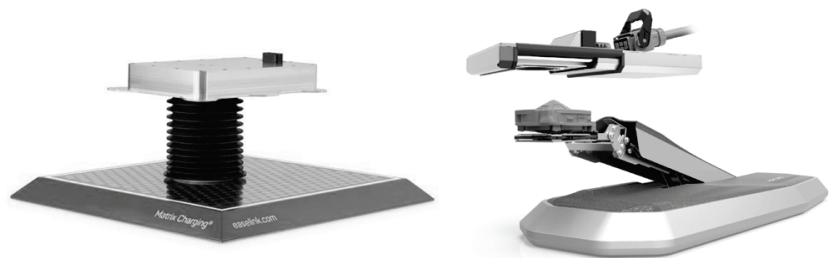

Figure 5 Underbody coupler charging system of Easelink [9], left, and Volterio [10], right

\subsubsection{Conductive Side Coupler}

Manual conductive side charging by use of cables and plugs is the most common method today. In automotive applications, different standardized interfaces are used, as exemplarily shown in Fig. 6.

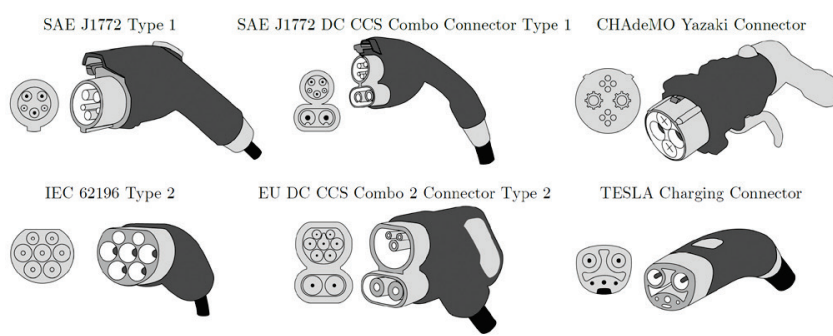

Figure 6 Selection of standardized charging interfaces in automotive applications [11]

Standardized interfaces provide several advantages, such as compatibility, certification for predefined charging voltage and current as well as broad availability of systems and components. In the present work, the Combined Charging System (CCS) Type 2 system [12] was considered in more details. This interface enables both AC and DC charging, and that with a wide power range of up to $30 \mathrm{~kW}$ for $\mathrm{AC}$ and up to $350 \mathrm{~kW}$ for $\mathrm{DC}$ at a voltage level of up to
$1000 \mathrm{~V}$. The interface design is standardized according to the IEC 61196 and the operational use is certified according to IP44. Besides transfer of energy, the interface also allows communication and data exchange, e.g. between the charging station and the vehicle according to the IEC 15118. In this way, the CCS Type 2 is not only applicable for automotive charging, but also for the use at commercial vehicles and in industrial facilities.

While conductive charging standard interfaces have been developed to provide a safe and reliable charging connection by manual operation, they have not been developed for automated robot-controlled plugging. This brings several challenges for the development of automated charging systems, because design details of the connectors hinder smooth automated plug-in and plug-out processes. Exemplary, pre-positioning of plug and socket is not supported by markers or lights, so that an automated charging device has to operate with highly accurate vision systems, able to deal with different surface- and light- conditions. In addition, the shapes of plug and socket are not optimized for automated fitting, e.g. by use of tapered or conical wall shapes that would support self-centering effects. Contrary, the walls are designed in parallel to the moving direction and do not support the fitting process. In this way, the development of an automated robot-controlled charging system represents a challenging task and requires to integrate a precise sensor system for position detection and control in combination with an accurate robotic actuation system for conduction of the necessary motion during the plug-in and plug-out sequences. Due to the high complexity, no serial production system has been introduced so far. However, different car manufacturer and supplier have published their research and development activities in this field during the past years, e.g. Volkswagen [13], Tesla [14], Kuka robotics [15]. Fig. 7 shows a selection of prototype systems for conductive side coupling.

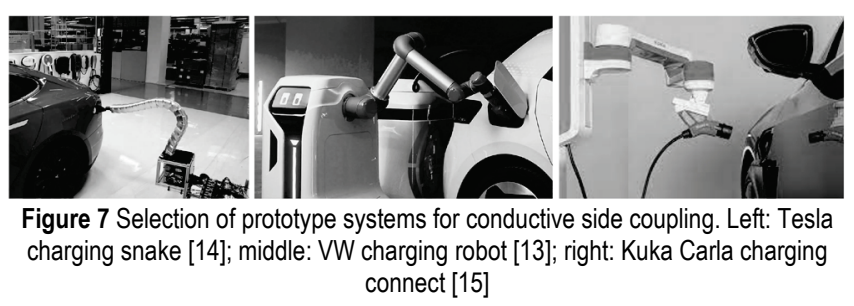

\section{AUTOMATED CONDUCTIVE CHARGING SYSTEM USING STANDARDIZED CONNECTORS}

The different charging technologies, introduced in section 2, have been evaluated regarding their potential use in large-scale industrial applications. Boundary conditions for the evaluation included:

- Compatibility with different types and sizes of electric vehicles

- Compatibility with both manual driven and fully autonomously operating vehicles

- Safety and reliability of the technology

- Easy operation and maintenance 
- Costs of installation, investment and service

- Availability and maturity of the technology.

As a result, the conductive charging technology with standardized interfaces reached the highest scores and has been selected for further consideration. Convincing strengths of this technology include the applicability for a fleet with very different types of vehicles (referred to Fig. $1 \& 2$ ), the possibility to use proven standardized interfaces from the automotive sector, low electrical losses and EMC in operation as well as high safety and reliability of the technology. In addition, state-of-the-art charging converters can be applied and installation, operation and maintenance can be conducted with reasonable effort. One critical aspect in the evaluation included the missing maturity of the technology: automated conductive coupling with standard interfaces is not available as a mass production product and thus beyond today's state-of-the-art.

In this context, this publication introduces results of a research project that focused on the development of a fully automated charging robot, using the CCS Type 2 standard interface [16-18]. The charging robot consists of a visionbased sensor system, a 6-axis robotic arm including a CCS plug as well as a system control unit.

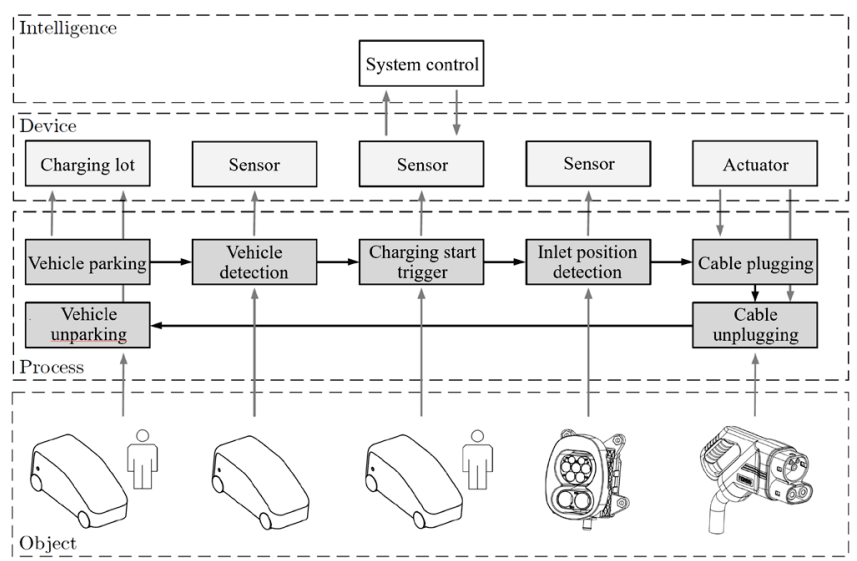

Figure 8 Overview of the automated conductive side coupler charging system

Fig. 8 shows the operational sequences of the automated charging process, including involved components. "Sensor" represents a camera system, "Actuator" the robotic arm and "System control" represents the station controller, including data communication interfaces. As a part of the charging process sequence, the "Objects" are represented by involved manually or automated controlled target vehicles, and in subsequent phases of the plugging process by the charging socket and the charging plug.

At the beginning of the charging process, the target vehicle approaches to the charging station and parks in a predefined position. Due to a given operating range of about 1 meter, different types of vehicles with different charging socket positions can be processed. It can be stated that the necessary parking position accuracy is relatively low in comparison with inductive or conductive underbody coupling. The charging system detects and identifies the vehicle on the spot by use of a camera-based object recognition system and allocates the specific vehicle characteristics from a database. In this way, vehicle charging requirements are set, e.g. actual position of the charging plug or electric parameters of the charging process. In the next step, the charging process is started; this can be done manually or fully automated - depending on the type of vehicle to be charged. In the first step, the charging plug is roughly aligned to the vehicle's charging socket and in a second step, precisely motion sequences perform very accurately the critical plug-in process. Both steps are controlled by use of a camera-based object detection and identification system [5]. After the battery is charged to a predefined level of State-of-Charge (SOC), the robotic system unplugs and the vehicle can leave the charging station. The entire procedure is recorded and key data are stored in a database system to support the vehicle fleet management.

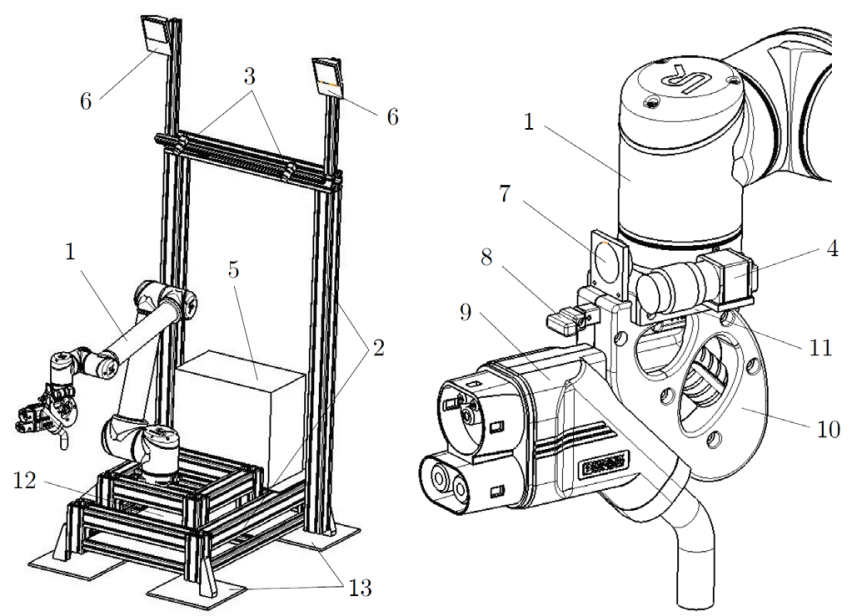

Figure 9 Left: overview of the charging station, right: connector unit with CCS Type 2 connector, camera, LED, actuator and adapters [5]

Fig. 9 shows the configuration of the automated charging station and the connector unit. The station consists of robot (1), frame (2), cameras $(3,4)$, robot control box (5), LEDs (6, 7), actuator (8), CCS Type 2 connector (9), two carrier adapters $(10,11)$, rubber damper (13) and a microcontroller board (frame-integrated, 12). The integrated robot (1) is a collaborative UR10-CB3, [19]. The 2D-cameras (3 and 4) are responsible for the identification of vehicle and inlet pose detection. Two LEDs (6) on the frame (2) and the LED (7) on the robot head tool support the vision system in case of insufficient light conditions. An additionally integrated actuator (8) enables closing of charging lids, if required. Two adapters, whereas one is made of aluminium (11), the other one (10) is made of 3D-printed plastics to reduce weight, improving robot control and cable handling, are integrated and hold the parts in a compact system that is connected with the robot. Besides this stationary configuration, the charging robot has been designed for mobile application, too (Fig. 11).

The movement of the robot head - and thus of the charging connector - results in a sum of sectional performed movements of the robotic system. The total accuracy of the movement is influenced by the accuracy of vision system, control and motion accuracy of the robot, as well as by mechanical deviation and tolerances of the components. 
Additionally, influencing factors include temperature extension, vibrations and environmental impacts. All these factors have to be considered in the system control to enable safe and reliable operation.
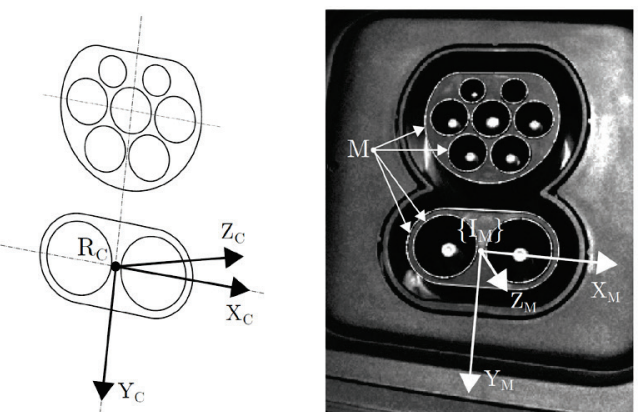

Figure 10 Left: CAD-model for shape-based 3D-matching, right: matching result of an image from the robot-head camera [5]

A specific challenge for successful operation represented the development of a highly-accurate position detection and object matching process. The matching process is based on a vision-based algorithm, using a CAD-model of the specific connector surface shape for processing in the vision software Halcon [20]. In this way, the position of the coordinate system of the shape model $\left(X_{\mathrm{C}}, Y_{\mathrm{C}}, Z_{\mathrm{C}}, R_{\mathrm{C}}\right)$ is aligned in relation to the position and orientation of the socked in the camera image $\left(X_{\mathrm{M}}, Y_{\mathrm{M}}, Z_{\mathrm{M}},\left\{I_{\mathrm{M}}\right\}\right)$. Fig. 10 shows the CADmodel including the pre-defined coordinate system and a positive matching result during tests.

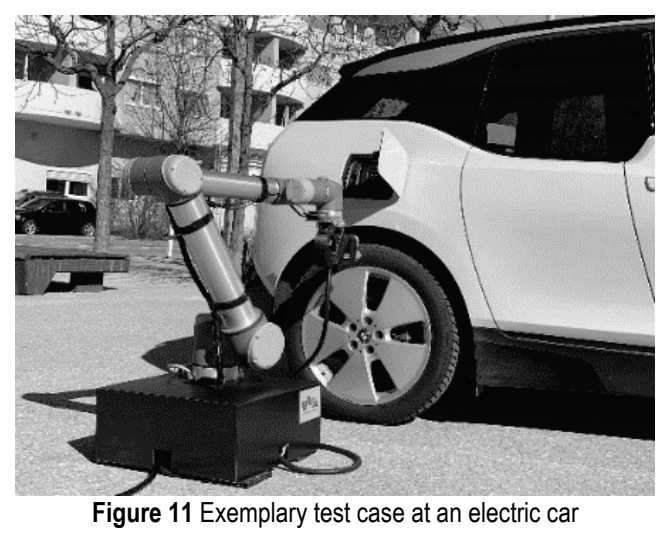

The automated charging station has been tested at different vehicles under various boundary conditions in buildings and outside. It was shown that the introduced technology enables safe and reliable plug-in and plug-out processes with a very high rate of success. In case of errors (which occurred in less than 1\% of the test cases), the system automatically goes back to the initial starting position and restarts the plug-in process. The robotic system can be combined with different types of electric charging stations, according to the actual charging performance requirements. Fig. 11 shows an exemplary test case of charging an electric personal car at a parking lot and the compact design of this charging robot version. In this context, the system is applicable in various positions and can be even mounted on movable platforms or robotic vehicles to charge a number of vehicles in a subsequent order by plugging-in the cable at on car and then driving to the next one.

\section{CONCLUSIONS}

Automated charging of electric vehicles in industrial environment represents an important upcoming technology to enable effective operation, safe and reliable charging processes and maximum comfort. Considering emerging autonomous vehicles, automated charging is mandatory to enable holistic management of fleets. A detailed study of the different electric charging technologies showed, that automated conductive charging by use of standardized charging interfaces has the greatest potential for successful implementation. In this context, the paper presents a novel automated robot-controlled charging station that is universally applicable for different charging standards and different types of vehicles. In the present work, the station has been equipped with the CCS Type 2 connector standard. However, the technology is not limited to this example and can also be equipped with different connector standards and also different main actuators (robots). A comprehensive process has been introduced, covering all sequences of the charging process, including approaching the vehicle to the charging station, detection and identification of vehicle and charging socket, automated plug-in process, charging sequence as well as automated plug-off and vehicle exit. In addition, communication between vehicle, charging station and data management supporting comprehensive fleet management applications, has been introduced. In this way, a system for autonomous charging of electric vehicles is provided that can significantly support service and management of vehicle fleets in different types of industrial environments.

\section{Acknowledgements}

The authors want to express their acknowledgement to the project partners BMW AG, MAGNA Steyr Engineering, KEBA AG, Austrian Society of Automotive Engineers (OEVK) and the Institute of Computer Science and Engineering at the University of South Florida (USF) for the excellent cooperation. In addition, thanks go to the Austrian Research Promotion Agency (FFG) and the Austrian Federal Ministry of Transport, Innovation and Technology (bmvit) for funding.

\section{Notice}

The paper will be presented at MOTSP $2021-12$ th International Conference Management of Technology - Step to Sustainable Production, which will take place in Poreč/Porenzo, Istria (Croatia), on September 8-10, 2021. The paper will not be published anywhere else.

\section{REFERENCES}

[1] Jungheinrich - Solutions for Intralogistics, https://www.jungheinrich.de/ (accessed: 2021.03.05) 
[2] Cleanfix professional cleaning equipment, https://www.cleanfix.com/ (accessed: 2021.03.05)

[3] Asti mobile robots, https://www.astimobilerobotics.com/ (accessed: 2021.03.05)

[4] Cleanfix Fully Automated Docking Station, https://www.ra660navi.com (accessed: 2021.03.05)

[5] Walzel, B. (2020). Automated Charging of Electric Vehicles with Conductive Standards. Doctoral thesis submitted at Graz University of Technology, Austria.

[6] BMW Wireless Charging, https://www.bmw.de/ (accessed: 2018.01.17)

[7] Squatriglia, C. (2009). Better Place Unveils an Electric Car Battery Swap Station. Wired, https://www.wired.com/ 2009/05/better-place/ (accessed: 2021.03.05)

[8] Change-Climate: Nio China - Battery Cartridge EV's, https://www.change-climate.com/Transport_Land_Sea_Su stainable/SMART_Hybrid_EV_Energy_Service_Networks/N IO_China_Chinese_Battery_Swap_Cartridge_Exchange_Stati ons_Cars.htm (accessed: 2021.03.05)

[9] Easelink Matrix Charging, https://easelink.com (accessed: 2021.03.05)

[10] Volterio Automatic Electric Charging, https://www.volterio.com, (accessed: 2021.03.05)

[11] EV-Institute: Plug-In Around the EV-World, https://evinstitute.com/images/ (accessed: 2018.01.17)

[12] Phoenix Contact, Technical data of Phoenix Contact Charging Equipment, https://www.phoenixcontact.com (accessed: 2020.02.02)

[13] Volkswagen AG: Volkswagen lets its charging robots loose, https://www.volkswagenag.com/en/news/stories/2019/12/ volkswagen-lets-its-charging-robots-loose.html\# (accessed: 2021.03.05)

[14] Tesla: Charger prototype finding its way to Model S, https://www.youtube.com/watch?v=uMM01RfX6YI (accessed: 2016.08.06)

[15] Kuka AG - Charging Assistant Carla Connect Charges Electric Cars, web-link: www.023jjzs.com/nezbb1/z7nzw3.html, (accessed: 2021.03.05)

[16] Walzel, B., Hirz, M., Brunner, H., \& Kreutzer, N. (2019). Robot-Based Fast Charging of Electric Vehicles. SAE Paper 2019-01-0869, e-ISSN: 2688-3627. https://doi.org/10.4271/2019-01-0869

[17] Miseikis, J., Ruther, M., Walzel, B., Hirz, M., \& Brunner, H. (2017). 3D vision guided robotic charging station for electric and plug-in hybrid vehicles. Cornell University, arXiv: 1703.05381 [cs.RO]

[18] Walzel, B., Sturm, C., Fabian, J., \& Hirz, M. (2016). Automated robot-based charging system for electric vehicles. The $16^{\text {th }}$ International Stuttgart Symposium Vehicle and Engine Technology, Germany. https://doi.org/10.1007/978-3-658-13255-2_70

[19] Universal Robots: Technical Data Universal Robot UR10, https://www.universal-robots.com (accessed: 2017.07.03)

[20] MVTEC. (2017). HALCON Version 13.0.1, Vision Software, MVTec Software GmbH, Munich, Germany.

\section{Authors' contacts:}

Mario Hirz, Associate Prof. Dr.

Institute of Automotive Engineering, Graz University of Technology, Inffeldgasse 11/2, $8010 \mathrm{Graz}$, Austria

E-mail: mario.hirz@tugraz.at

Bernhard Walzel, Dr. Dipl.-Ing.

Institute of Automotive Engineering, Graz University of Technology, Inffeldgasse 11/2, $8010 \mathrm{Graz}$, Austria

E-mail: bernhard.walzel@tugraz.at

Helmut Brunner, Dipl.-Ing.

Institute of Automotive Engineering, Graz University of Technology, Inffeldgasse 11/2, $8010 \mathrm{Graz}$, Austria

E-mail: helmut.brunner@tugraz.at 